\title{
Efeitos da Eletroestimulação Funcional e Kabat na Funcionalidade do Membro Superior de Hemiparéticos
}

\author{
Effects of functional electrical stimulation and Kabat on functionality of the upper limb of hemiparetic
}

Priscilla da Silva Marques ${ }^{1}$, Scheyla Paula Bollmann Oleskovicz Nogueira²

\begin{abstract}
RESUMO
Objetivo. Analisar a utilização de eletroestimulação funcional (EEF) associado ao método Kabat no desempenho da capacidade funcional do membro superior parético no portador de sequela motora decorrente de acidente vascular cerebral (AVC) na fase crônica (até 6 meses). Método. Foram selecionados 2 pacientes aleatoriamente, sendo que o primeiro recebeu tratamento por meio da EEF e método Kabat (diagonal D2) simultaneamente e o segundo paciente selecionado recebeu tratamento somente através da EEF. Nas avaliaçôes $\left(1^{\circ}, 10^{\circ}\right.$ e $20^{\circ}$ atendimento e 30 dias após o término do tratamento) do membro superior parético utilizou-se a Escala de funcionalidade de Fugl Meyer, Escala de Ashworth Modificada para avaliação do tônus muscular e Índice de Barthel para avaliação das atividades de vida diária. Resultados. Houve melhora da movimentação passiva, dor, sensibilidade e função motora em ambos os pacientes apesar das diferenças funcionais entre os mesmos; redução do tônus muscular e melhora nas atividades de vida diária. Sendo que, os ganhos mantiveram-se 30 dias após o término da intervenção. Conclusáo. Os dois procedimentos (Kabat e EEF; somente EEF) se mostraram eficientes para o ganho de capacidade funcional do membro superior parético, viabilizando uma nova alternativa de tratamento.
\end{abstract}

Unitermos. Acidente Vascular Cerebral, Estimulação Elétrica, Extremidade Superior.

Citação. Marques PS, Nogueira SPBO. Efeitos da Eletroestimulação Funcional e Kabat na Funcionalidade do Membro Superior de Hemiparéticos.

\begin{abstract}
Objective. Analyze the use of functional electrical stimulation (FES) method associated with the Kabat performance of the functional capacity of upper limb in the carrier for motor sequel resulting from stroke in chronic phase (up to 6 months). Method. 2 patients were selected randomly, with the first received treatment by the FES and Kabat method (diagonal D2) while the second patient was treated only selected by FES. In the evaluations $\left(1^{\circ}, 10^{\circ}\right.$ and $20^{\circ}$ service and 30 days after completion of treatment) of the upper limb used the Scale feature Fugl Meyer, Modified Ashworth Scale for assessment of muscle tone and Barthel Index for assessing the activities daily life. Results. Improvement of passive movement, pain sensitivity and motor function in both patients despite the functional differences between them, reducing muscle tone and improvement in activities of daily living. Since the gains were maintained 30 days after the end of the intervention. Conclusion. The two procedures (Kabat and FES; only FES) has proved effective to gain the functional capacity of upper limb, allowing a new treatment alternative.
\end{abstract}

Keywords. Stroke, Electric Stimulation, Upper Extremity.

Citation. Marques PS, Nogueira SPBO. Effects of functional electrical stimulation and Kabat on functionality of the upper limb of hemiparetic.
Trabalho realizado na Faculdade Estácio de Sá de Santa Catarina, Sáo JoséSC, Brasil.

1. Acadêmica da nona fase do Curso de Fisioterapia da Faculdade Estácio de Sá de Santa Catarina, São José-SC, Brasil.

2. Fisioterapeuta, Mestre Professora Supervisora do Estágio de Fisioterapia Neurofuncional da Faculdade Estácio de Sá de Santa Catarina, FlorianópolisSC, Brasil.
Endereço para correspondência:

Priscilla da Silva Marques

R. Tereza Cristina, 380 CEP 88070-790, Florianópolis-SC, Brasil. E-mail: priscillam_fisio@hotmail.com

Relato de Caso Recebido em: 06/07/10 Aceito em: 11/04/11

Conflito de interesses: não 


\section{INTRODUÇÃO}

O Acidente Vascular Cerebral (AVC) consiste em uma manifestação, muitas vezes súbita, em que ocorre a interrupção do suprimento sanguíneo cerebral que pode provocar lesão celular e alteraçôes nas funções neurológicas. Dentre as causas do acidente vascular cerebral encontram-se a hipertensão arterial, hiperlipidemia, diabetes, malformação dos vasos sanguíneos, tumores cerebrais, traumas e outras condiçóes ${ }^{1}$.

O AVC é a principal causa de incapacidade neurológica dependente de cuidados de reabilitação, levando a comprometimentos da função motora, cognitiva, sensorial, psicológica e social.

O membro superior parético do indivíduo com sequela de AVC limita suas atividades motoras, desde a mais simples até a mais complexa. Essas restrições são consequências dos prejuízos relacionados à alteração do tônus, força muscular, amplitude de movimento e habilidades motoras específicas para o membro referido, podendo levar o paciente a uma deterioração de dimensão psicossocial $^{2}$.

Uma das técnicas utilizadas na reabilitação após o AVC é a Estimulação Elétrica Funcional (EEF), que pode apresentar-se como um auxílio na produção de contração funcionalmente útil de um músculo privado de controle normal, permitindo ativar não só a musculatura local, mas também mecanismos reflexos necessários à reorganização da atividade motora ${ }^{3}$. Do mesmo modo, o método Kabat (Facilitação Neuromuscular Proprioceptiva) visa conseguir o movimento normal que dependerá das açóes integradoras do sistema nervoso central, da morfologia, da cinesiologia, do aprendizado do desenvolvimento motor e da conduta motora; apresentando padróes básicos de movimento que promovem e aceleram as respostas dos mecanismos neuromusculares através da estimulação dos receptores ${ }^{4}$.

Em vista disso, apesar da possibilidade de benefícios do uso da EEF nos membros superiores de pacientes hemiparéticos, esse recurso tem tido uso limitado na clínica, o que pode ser atribuído à falta de conhecimento dos efeitos e parâmetros adequados de estimulação. Sendo assim, diante da questáo clínica relativa aos efeitos da EEF pós AVC, o objetivo deste estudo foi analisar a utilização de EEF associado ao método Kabat no desempe- nho da capacidade funcional do membro superior parético no portador de sequela motora decorrente de acidente vascular cerebral na fase crônica (até 6 meses pós AVC).

\section{MÉTODO}

\section{Amostra}

A amostra foi selecionada por um processo de amostragem aleatória intencional (não-probabilística), onde selecionou-se, por meio da lista de espera de pacientes da Clínica de Fisioterapia da Faculdade Estácio de Sá de Santa Catarina, 2 indivíduos, do sexo masculino com diagnóstico de acidente vascular cerebral isquêmico, com idade superior a 50 anos, que residem na grande Florianópolis.

\section{Procedimento}

Após a seleção e contato por telefone com os pacientes, a intervenção ocorreu na Clínica de Fisioterapia da Faculdade Estácio de Sá de Santa Catarina, sendo que, o primeiro indivíduo selecionado recebeu tratamento com aplicação de EEF e método Kabat (diagonal D2 - padrão extensão-adução e rotação interna passando para padrão flexão, abdução e rotação externa) e o segundo indivíduo foi contemplado apenas pela EEF.

O tratamento fisioterapêutico consistiu em 3 sessóes semanais, com intervalo de 1 dia entre elas e duração de 40 minutos, sendo realizado um total de 20 atendimentos.

Foram aplicados os seguintes instrumentos respectivamente no $1^{\circ}, 10^{\circ}, 20^{\circ}$ atendimento e 30 dias após o término da intervenção: Escala Fugl Meyer utilizada para avaliar comprometimento motor e sensorial do membro superior acometido seguido ao AVC. Foi desenvolvida e introduzida, em 1975, por Fugl-Meyer e colaboradores5. Esta escala foi o primeiro instrumento quantitativo para mensuração sensório-motora da recuperação do AVC e é, provavelmente, a escala mais conhecida e usada para a pesquisa e/ou prática clínica ${ }^{6}$. Foram utilizados os seguintes itens da Escala Fugl Meyer: I (movimentação passiva e dor no membro superior), II (sensibilidade do membro superior), III (funçáo motora do membro superior) e IV (coordenação e velocidade do membro superior). Escala de Ashworth Modificada para avaliação do tônus muscular de flexores de cotovelo. Esta escala é a ferramenta mais utilizada na clínica semiológica pelo Fisioterapeuta, apesar de sua reconhecida subjetividade? ${ }^{7}$ Índice de Barthel 
para avaliação das atividades de vida diária (AVD'S), o qual possui os domínios de alimentação, higiene pessoal, vestir-se, controle da bexiga, do intestino, deambulação, subir escadas, transferência da cadeira para cama. A pontuação é de zero, cinco, dez e quinze; a nota é proporcional à independência, quanto maior for a nota mais independente é o indivíduo. A pontuação máxima é 100 e, abaixo de 50 significa dependência ${ }^{8}$. As reavaliaçóes foram realizadas no $10^{\circ}$ e $20^{\circ}$ atendimento e após 30 dias da última intervenção, onde os mesmos instrumentos foram aplicados novamente no mesmo local, para comparar as variáveis observadas. Os parâmetros da eletroestimulação (EEF) em ambos os pacientes foram: Frequência = $20 \mathrm{~Hz}$; Ciclo on $=6$ segundos; ciclo off $=12$ segundos; rise $=3$ segundos e decay $=3$ segundos ${ }^{3}$. A intensidade foi aumentada conforme a tolerância do paciente, atingindo em torno de $4 \mathrm{~mA}$, no modo sincronizado. Os eletrodos foram posicionados nos pontos motores dos músculos supra-espinhoso, deltóide posterior, tríceps braquial e extensores de punho e dedos. Na primeira semana de atendimento os pacientes foram submetidos a $15 \mathrm{minu}-$ tos de estimulação; na segunda semana a 20 minutos; na terceira semana a 25 minutos e o restante dos atendimentos (11) o tempo total de estimulação foi de 30 minutos. Essa progressão de tempo foi realizada a fim de que não ocorresse fadiga muscular.

\section{Relato dos Casos}

O primeiro paciente selecionado para o tratamento, contemplado por EEF e método Kabat, foi D. J. da C., 66 anos, gênero masculino, aposentado, com diag- nóstico clínico de AVC isquêmico. Sofreu AVC na data de 11/12/2009 e como sequela, paciente apresenta hemiplegia esquerda.

O segundo paciente selecionado, contemplado apenas por EEF, foi L. M. da R., 73 anos, gênero masculino, aposentado, com diagnóstico clínico de AVC isquêmico. Sofreu AVC na data de 23/12/2009 e como sequela, paciente apresenta hemiparesia esquerda.

Ambos os pacientes possuíam 3 meses pós-AVC quando foi dado início à coleta de dados da presente pesquisa. Sendo esta coleta realizada no período de março à junho de 2010.

\section{RESULTADOS}

Observa-se que houve melhora da movimentação passiva, dor, sensibilidade e função motora em ambos os pacientes. Na subescala coordenação e velocidade de movimento do membro superior, somente o paciente 2 (EEF) obteve ganhos na pontuaçãoo (Tabela 1).

A pontuação máxima da função motora da extremidade superior é igual a sessenta e seis pontos (66) e a da inferior, trinta e quatro (34), resultando em uma pontuação da função motora máxima igual a cem pontos (100), o que define a função motora normal ${ }^{9}$. Foi estabelecido ainda, quatro níveis de comprometimento motor: nível severo corresponde a pontuaçóes menores que 50, que representam pequena ou nenhuma movimentaçáo voluntária dos membros afetados; nível marcante com pontuação de 51 a 84; nível moderado com pontuação entre 85 a 95, e nível leve de comprometimento motor quando a pontuação ocorre de 96 a $99^{9}$.

\section{Tabela 1}

Pontuação obtida através da avaliação de Desempenho Físico de Fugl Meyer

\begin{tabular}{|c|c|c|c|c|c|c|c|c|c|c|c|c|c|c|c|c|c|c|c|c|}
\hline \multirow[t]{3}{*}{ PACIENTES } & \multicolumn{20}{|c|}{ SUBESCALAS } \\
\hline & \multicolumn{4}{|c|}{$\begin{array}{c}\text { Movimentaçáo } \\
\text { Passiva } \\
(\mathbf{P M = 2 4 )}\end{array}$} & \multicolumn{4}{|c|}{$\begin{array}{c}\text { Dor } \\
(\mathrm{PM}=24)\end{array}$} & \multicolumn{4}{|c|}{$\begin{array}{c}\text { Sensibilidade } \\
(P M=14)\end{array}$} & \multicolumn{4}{|c|}{$\begin{array}{c}\text { Funçáo Motora } \\
\text { do MS } \\
(\mathrm{PM}=60)\end{array}$} & \multicolumn{4}{|c|}{$\begin{array}{c}\text { Coordenaçáo/ } \\
\text { Velocidade } \\
\text { MS } \\
(\mathrm{PM}=6)\end{array}$} \\
\hline & 1 & 2 & 3 & 4 & 1 & 2 & 3 & 4 & 1 & 2 & 3 & 4 & 1 & 2 & 3 & 4 & 1 & 2 & 3 & 4 \\
\hline Paciente 1 & 23 & 24 & 24 & 24 & 2 & 2 & 24 & 24 & 10 & 11 & 11 & 11 & 7 & 11 & 11 & 11 & 3 & 3 & 3 & 3 \\
\hline Paciente 2 & 23 & 24 & 24 & 24 & 1 & 1 & 24 & 24 & 10 & 12 & 12 & 12 & 36 & 56 & 60 & 60 & 3 & 5 & 5 & 5 \\
\hline
\end{tabular}

PM: Pontuação máxima; 1: Primeira avaliação; 2: Segunda avaliação; 3: Terceira avaliação; 4: Quarta avaliação; Fonte: Dados primários, 2010. 
De acordo com o exposto, para graduar os níveis de severidade somente da função motora do membro superior, foi subtraído 34 pontos referentes ao membro inferior dos valores de nível de severidade citados acima. Assim, foi encontrado os seguintes valores: nível severo de função motora do membro superior corresponde a pontuaçóes menores que 16; nível marcante com pontuação de 17 a 50; nível moderado com pontuação entre 51 a 61, e nível leve de comprometimento motor do membro superior quando a pontuação atingir 62 a 65 .

O paciente 1 (EEF e Kabat) atingiu pontuação de 7 pontos na primeira avaliação e 11 pontos nas últimas 3 avaliaçôes, o que configura-o com um nível severo de função motora. O paciente 2 (EEF) obteve uma pontuação de 36 na primeira avaliação, indicando nível marcante de função motora; 56 pontos na segunda avaliação, com nível moderado de função motora do membro superior; e 60 pontos na terceira e quarta avaliação, que remete-o também ao nível moderado de função motora do membro superior (Gráfico 1).

Com relação ao tônus muscular avaliado através da Escala de Ashworth Modificada foi observado que houve redução do tônus do membro superior parético em ambos os pacientes (Gráfico 2).

Para o paciente 1, observa-se que na primeira avaliação através do Índice de Barthel, o escore total foi de 80 , representando uma dependência leve. Sua pontuação dos domínios, nas 4 avaliaçôes foram respectivamente:
10-10-10-10 (Alimentação); 0-5-5-5; (Banho); 5-5-5-5 (Vestuário); 10-10-10-10 (Controle de intestino); 10-1010-10 (Controle de bexiga); 10-10-10-10 (Locomoção até o banheiro); 10-10-10-10 (Transferências); 10-1010-10 (Mobilidade e deambulação); 10-10-10-10 (Subir escadas). Na segunda, terceira e quarta reavaliação, o paciente conseguiu escore de 85 pontos, ainda mantendo uma dependência leve, pois somente no domínio banho tornou-se independente (Gráfico 3).

Para o paciente 2 , observa-se que na primeira avaliação o escore total foi de 70, representando uma dependência leve. Sua pontuação dos domínios, nas 4 avaliaçôes foram respectivamente: 5-10-10-10 (Alimentação); 0-55-5 (Banho); 5-5-5-5 (Vestuário); 5-5-5-5- (Toalete) 1010-10-10 (Controle de intestino); 10-10-10-10 (Controle de bexiga); 10-10-10-10 (Locomoção até o banheiro); 5-15-15-15 (Transferências); 10-15-15-15 (Mobilidade e deambulação); 10-15-15-15 (Subir escadas). Na segunda, terceira e quarta reavaliação, o paciente atingiu escore de 100 pontos, tornando-o independente. Sendo que alcançou a independência nos domínios alimentação, banho, locomoção, transferências, mobilidade e deambulação, subir escadas (Gráfico 4).

\section{DISCUSSÃO}

A espasticidade gera mudanças morfológicas, fisiológicas e biomecânicas nos músculos, causando dor e diminuição da amplitude de movimento ${ }^{10}$. Desta forma,

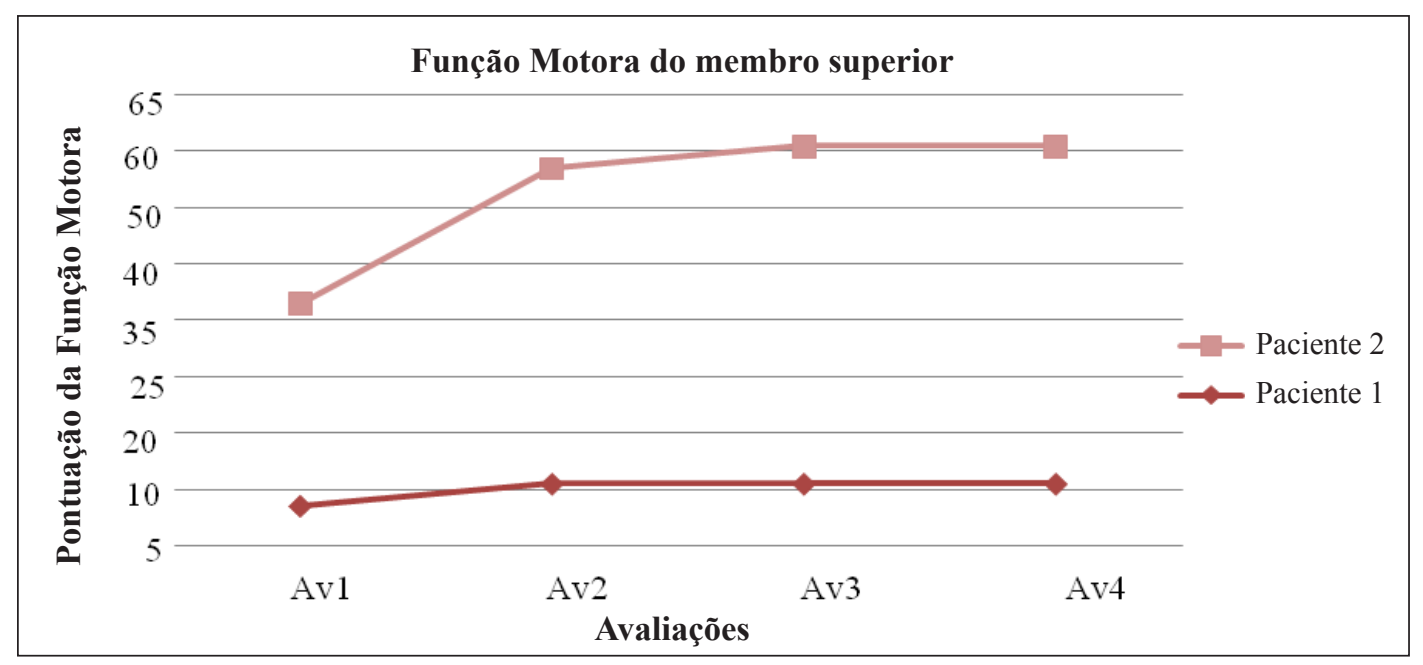

Gráfico 1. Função motora do membro superior em cada paciente nas 4 avaliaçóes.

Av1: Primeira avaliação; Av2: Segunda avaliação; Av3: Terceira avaliação; Av4: Quarta avaliação. 


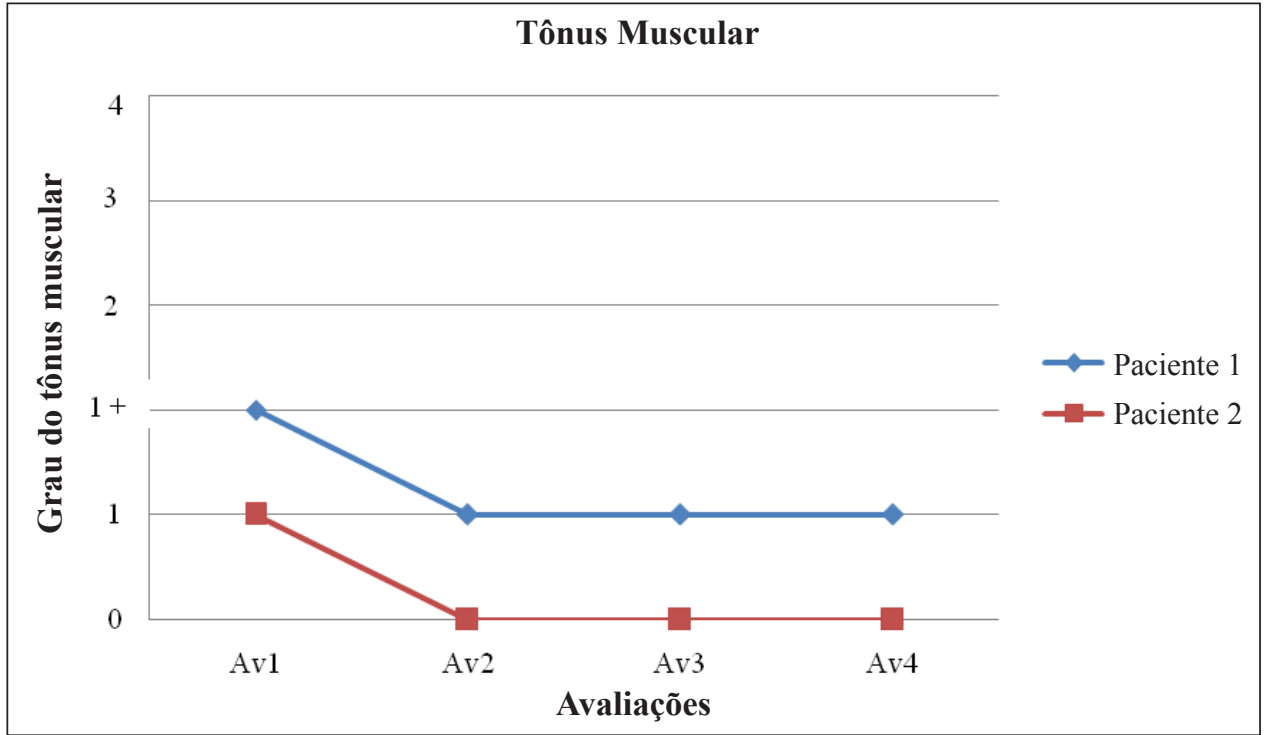

Gráfico 2. Evolução do tônus muscular nos pacientes. no paciente 2 que recebeu tratamento apenas por meio da EEF. No entanto, os valores observados ao final do estudo no paciente 1 que recebeu tratamento através da EEF e Kabat, ainda eram insuficientes para tornar a extremidade superior funcionalmente ativa. Esses dados assemelham-se aos encontrados em um estudo que analisou aspectos preditivos da recuperação motora de pacientes hemiplégicos, onde observou-se que mais a EEF foi determinante para a diminuiçáo da dor e melhora da movimentação passiva em ambos os pacientes, pois ocorrendo a contraçáo do antagonista ao músculo espástico, acredita-se que há uma melhora da espasticidade do músculo agonista levando, assim, à reestruturação do ato motor no hemiplégico e redução da dor ${ }^{11}$. Da mesma forma, o método Kabat contribuiu para a melhora da movimentaçáo passiva, visto que trabalha os receptores sensoriais que enviam informações relacionadas ao movimento e posicionamento corporal ${ }^{12}$.

Em termos quantitativos observou-se uma melhora expressiva da recuperação motora da extremidade superior, durante o período do estudo, principalmente

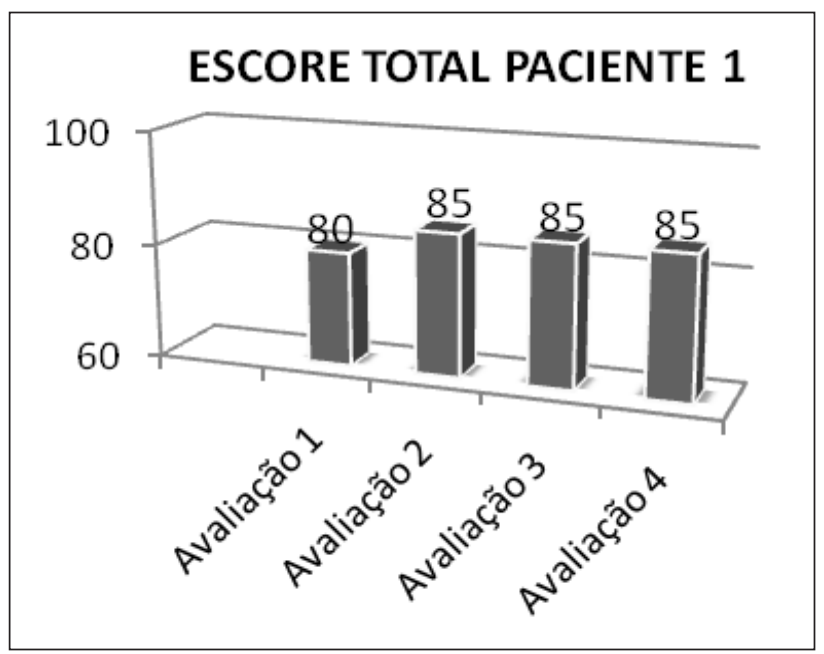

Gráfico 3. Escore total do Índice de Barthel nas avaliaçóes do paciente 1 . de $85 \%$ dos pacientes apresentavam déficits iniciais na extremidade superior, e após 3 a 6 meses, os problemas permaneciam em $55-75 \%$ dos pacientes ${ }^{13}$.

Muitos autores têm relatado que a estimulação elétrica funcional de um músculo aumenta sua força, diminui a espasticidade da musculatura antagonista e aumenta as amplitudes dos movimentos articulares ${ }^{14,15,16}$. E vários mecanismos estáo envolvidos para que essa reduçáo de espasticidade ocorra, dentre eles, o mais citado na literatura é o princípio da inervação e inibição recíprioca, onde as fibras Ia usam vias colaterais, estabelecendo sinapses facilitatórias, ao mesmo tempo em que fazem sinapse inibitória com a musculatura antagonista, na intenção de

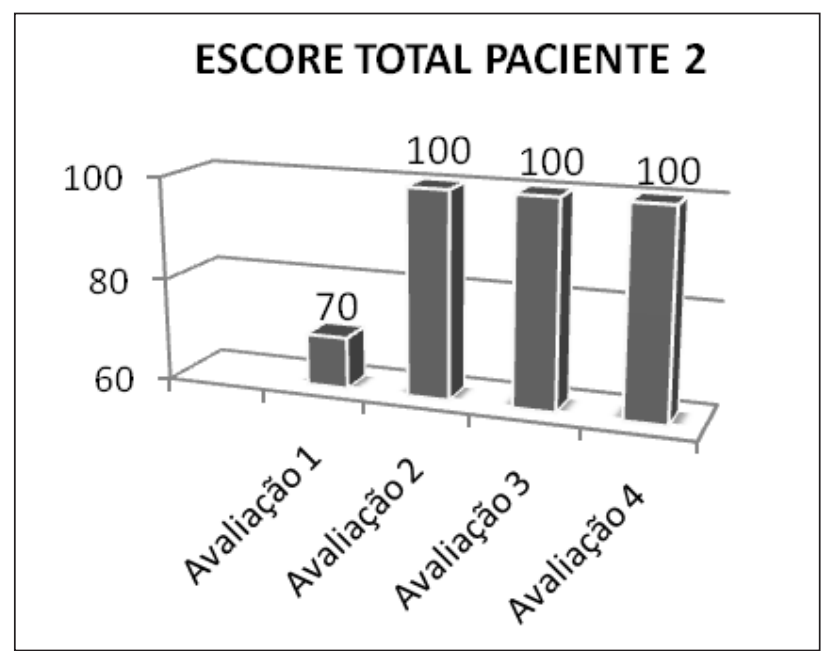

Gráfico 4. Escore total do Índice de Barthel nas avaliações do paciente 2 . 
promover relaxamento desta, possibilitando assim, uma contração adequada da musculatura agonista ${ }^{17}$. Vários autores reafirmam esta importância, relatando que, através da eletroestimulação funcional, há uma diminuição do tônus da musculatura antagonista ${ }^{18}$. Os achados do presente estudo confirmam os trabalhos supracitados, pois verifica-se a efetividade da eletroestimulação na redução da espasticidade dos pacientes atendidos nessa pesquisa.

Estudos mostram que ocorrem ganhos funcionais na rotina diária após EEF.

Há relatos de ganhos na destreza e funcionalidade na vida diária no grupo que recebeu EEF em comparação com o controle, o qual não foi contemplado pela eletroestimulaçãa ${ }^{19}$. No estudo em questão houve ganhos em relação às atividades da vida diária no paciente 2 (EEF), o qual apresentava menor comprometimento motor da extremidade superior. Esta variável pôde ser comprovada pelo Índice de Barthel. Porém, o paciente 1 (EEF e Kabat) alcançou a independência apenas no item banho, mantendo o escore da avaliação mesmo após os 20 atendimentos, o que pode ser atribuído ao seu maior comprometimento. O Índice de Barthel tem sido amplamente utilizado na monitoração das alteraçôes funcionais em indivíduos que estejam recebendo reabilitação. Embora não tenham sido completamente avaliadas suas propriedades psicométricas, o Índice de Barthel tem atingido elevadas correlações com outras medidas de incapacidade física ${ }^{20}$. Uma pontuação de 60 no Índice de Barthel foi fundamental para determinar a conquista da independência assistida. Os pacientes com AVC que obtiveram pontuação abaixo desse nível apresentaram acentuada dependência, enquanto as pontuaçóes inferiores a 40 corresponderam à dependência grave. Esses pacientes tipicamente tiveram estadas mais longas de reabilitação ${ }^{21}$. Os pacientes do presente estudo alcançaram pontuação que os remete a uma dependência assistida leve e à independência após a intervenção e após 30 dias do término do tratamento.

A estimulação dos grupos extensores de punho e dedos em um paciente hemiplégico reduz a espasticidade dos antagonistas flexores, e através da produção de movimentos extensores evocados repetitivos conseguese a manutenção do ganho funcional mesmo depois de cessada a aplicação do estímulo ${ }^{22}$. Esta afirmação pode ser confirmada, pois no estudo em questão ambos os pa- cientes mantiveram o ganho funcional mesmo após 30 dias de cessado o estímulo. Durante a análise da EEF na melhora da motricidade e função de membro superior em pacientes com AVC em fase aguda, percebeu-se que a EEF aumenta a recuperação motora do membro superior desses indivíduos ${ }^{23}$, o que entra em conformidade com achados da pesquisa em questão. Outro estudo, também analisou a resposta da EEF nos músculos extensores de punho, para se obter melhora da função motora de membro superior de pacientes pós-AVC e concluíram que a EEF melhora significantemente a função da mão e a atividade extensora do punho ${ }^{24}$.

Esta variável não pôde ser observada no paciente 1 (EEF e Kabat) do presente estudo, visto que o mesmo não obteve retorno da função de punho e mão, com ausência de movimento voluntário desses segmentos. Porém o paciente 2 (EEF) recuperou a função motora de punho e mão.

Um estudo investigou os efeitos durante um posicionamento do padráo da técnica do método Kabat comparada com uma posição neutra para o início de movimentos voluntários dos músculos do membro superior, através da avaliação dos potenciais motores evocados (PME) dos músculos braquiorradial e tríceps braquial. Os autores verificaram um aumento da amplitude do potencial motor evocado e diminuição da latência dos movimentos realizados a partir de padróes da técnica de Kabat comparado à posiçóes neutras, os autores referem que esses resultados são devidos ao fato de que a quantidade de estímulo sensorial que vem da periferia é maior na posição do método Kabat comparada a posição neutra, o que induz a mudanças no nível de excitação da área motora cortical e dos motoneurônios correspondentes, estimulando a neuroplasticidade ${ }^{25}$. Em vista disso, o paciente 1 contemplado por EEF e método Kabat pôde receber maior quantidade de estímulo sensorial, no entanto, seus ganhos motores e funcionais não foram significativos. Vários autores afirmaram que nenhum dos experimentos pesquisados por eles que tratavam dos resultados de diversos métodos fisioterapêuticos aplicados à hemiplegia e hemiparesia, comprovou que o método Kabat foi mais eficiente na recuperação da função motora do que a cinesioterapia convencional ${ }^{26}$. Um estudo que comparou a utilização da Estimulação Elétrica Funcional (EEF) e Kabat, na melhora da habilidade funcional da 
articulação do ombro em pacientes hemiparéticos após AVC, chegaram a conclusão que o método Kabat oferece melhores resultados na melhora da habilidade motora do que a EEF, quando aplicadas em pacientes com $\mathrm{AVC}^{27}$. Esta afirmação entra em contradiçẫo com o presente estudo, onde o paciente submetido aos 2 métodos (Kabat e eletroestimulação) obteve menores ganhos em relação ao paciente que somente recebeu eletroestimulação e adquiriu função motora e habilidade funcional. Porém, devese ressaltar que o paciente 1 (EEF e Kabat) apresentava maior comprometimento motor e por isso, talvez, não pôde adquirir maior recuperação funcional e motora.

\section{CONCLUSÃO}

Para o paciente neurológico, recuperar a integridade do membro superior é um fator decisivo, pois a funcionalidade dos membros superiores confere ao indivíduo o caráter de independência.

Sendo assim, após a análise dos dados pôde-se observar que houve melhora da capacidade funcional do membro superior parético em ambos os pacientes, apesar das diferenças funcionais entre os mesmos. As duas técnicas (Kabat e EEF; somente EEF) se mostraram eficientes para o ganho de capacidade funcional, diminuição do tônus muscular do membro superior parético e melhora na habilidade de realizar as atividades de vida diária. Isso nos remete a uma nova alternativa de tratamento nesses pacientes, visto que os ganhos motores mantiveram-se após 30 dias do término do tratamento.

A pequena amostra coíbe este estudo de colocar os achados aqui expostos como sendo fatos constantes desta população, mas apresenta, certamente, uma contribuição na área de pesquisa na reabilitação do hemiplégico.

\section{REFERÊNCIAS}

1.Rowland Lewis PM. Tratado de neurologia. 10. ed. Rio de Janeiro: Guanabara Koogan, 2000, 887p.

2.Trevisan CM. Efeitos da imagem mental na reabilitação da função motora do membro superior na hemiplegia após acidente vascular encefálico (Tese). Brasília: Universidade de Brasília, 2007, 129p.

3.Agne JE. Eletrotermoterapia: teoria e prática. Orium: Santa maria, 2004, $365 \mathrm{p}$.
4.Adler SS, Beckers D, Buck M. PNF: facilitação neuromuscular proprioceptiva. São Paulo: Manole, 1999, 401p.

5.Maki T, Quagliato EMABM, Cacho EWA, Paz LPS, Nascimento NH, Inoue MMEA, et al. Estudo de confiabilidade da aplicaçâo da escala de Fugl-meyer no Brasil. Rev bras fisioter 2006;10:177-83.

6.Gladstone DJ, Daniells CJ, Black SE. The Fugl-Meyer Assessment of motor recovery after stroke: a critical review of its measurement properties. Neurorehabil Neural Repair 2002;16:232-40.

http://dx.doi.org/10.1177/154596802401105171

7.Minutoli VP, Delfino M, Freitas STT de, Tortoza C. Efeito do movimento passivo contínuo isocinético na hemiplegia espástica. Acta fisiatr 2007;14:142-8. 8.Índice de Barthel (endereço da internet). São Paulo: Júnior. (última atualização 01/2008; citado em: 08/2007). Disponível em: http://www.medicinageriatrica.com.br/2007/01/08/saude-geriatria/indice-de-barthel/.

9.Cacho EWA, Melo FRLV de, Oliveira R de. Avaliação da recuperação motora de pacientes hemiplégicos através do protocolo de desempenho físico FuglMeyer. Rev Neurocienc 2004;12:94-102.3.

10.Dean CM, Mackey FH, Katrak P. Examination of shoulder positioning

After stroke: A randomized controlled pilot trial. Austr J Physiother 2000;46:35-46.

11. Miyazak EG, Rosa T da S, Nascimento APH, Oberg TD. Influência da estimulação elétrica funcional para adequação de tono muscular e controle motor em hemiplégicos. Intellectus - Revista Acadêmica Digital do Grupo POLIS Educacional 2008;4:1-11.

12.Facilitação neuromuscular proprioceptiva (FNP) na dor em ombro hemiparético após acidente vascular encefálico (AVE). Cascavel: Peres. (última atualização: 11/2006; citado em: 11/2006). Disponível em: http://www.fag.edu.br/ tcc/2006/Fisioterapia/facilitacao_neuromuscular_proprioceptiva_(fnp)_na_ dor_em_ombro_hemiparetico_apos_acidente_vascular_encefalico_(ave).pdf. 13. Olsen TS. Arm and leg paresis as outcome predictors in stroke rehabilitation. Stroke 1990;21:247-51.

http://dx.doi.org/10.1161/01.STR.21.2.247

14.Kitchen S, Bazin S. Eletroterapia: Prática baseada em evidências. 11. ed. São Paulo: Manole, 2003, 348p.

15.Low J, Reed A. Eletroterapia explicada: princípios e prática. 3. ed. São Paulo: Manole, 2001, 427p.

16.Soares AV, Pagliosa F, Oliveira GO. Estudo comparativo entre a estimulação elétrica neuromuscular de baixa e média frequência para o incremento da força de preensão em indivíduos sadios não-treinados. Fisioterapia Brasil 2002;3:345-50.

17.Eletroestimulaçấo funcional no controle da espasticidade em paciente hemiparético. São Paulo: Santana. (última atualização: 08/2009; citado em: 03/2009). Disponível em: http://www.fafibe.br/revistasonline/arquivos/revistafafibeonline/sumario/9/19042010083217.pdf.

18.Martins FLM, Guimarâes LHCT, Vitorino DFM, Souza LCF. Eficácia da eletroestimulação funcional na amplitude de movimento de dorsiflexão de hemiparéticos. Rev Neurocienc 2004;12:103-9.

19.Kimberley TJ, Lewis SM, Auerbach EJ, Dorsey LL, Lojovich JM, Carey JR. Electrical stimulation driving functional improvements and cortical changes in subjects with stroke. Exp Brain Res 2004;15:450-60.

http://dx.doi.org/10.1007/s00221-003-1695-y

20. Oliveira APR de, Freitas AM de. Efeitos da intervençáo fisioterapêutica nas habilidades funcionais e no equilíbrio de uma paciente com ataxia espinocerebelar: estudo de caso. Revista Fisioterapia e pesquisa 2006;13:53-9.

21.Granger CV, Dewis LS, Peters NC, Sherwood CC. Stroke rehabilitation: analysis of repeated Barthel Index measures. Arch Phys Med Rehabil 1979;60:7-14.

22.Estimulaçấo elétrica funcional aplicada a pacientes hemiplégicos:um pano- 
rama (endereço da internet) São Paulo: Nogueira e Lima. (última atualização: 02/2002; citado em: 02/2002). Disponível em: http://telemedicina.unifesp.br/ pub/SBIS/CBIS2002/dados/arquivos/319.pdf.

23. Chae J, Beathoux F, Bohinc T, Dobos L, Davis T, Friedl A, et al. Neuromuscular stimulation for upper extremity motor and functional recovery in acute hemiplegia. Stroke 1998;29:975-9.

http://dx.doi.org/10.1161/01.STR.29.5.975

24. Wright PA, Granat MH. Therapeutic effects of functional electrical stimulation on the upper limb of eight children with cerebral palsy. Developmental medicine \& child neurology 2000;42:724-7.

http://dx.doi.org/10.1111/j.1469-8749.2000.tb00033.x
http://dx.doi.org/10.1017/S0012162200001341

25.Shimura K, Kasai T. Effects of proprioceptive neuromuscular facilitation on the initiation of voluntary movement and motor evoked potentials in upper limb muscles. Rev Human Movement Science 2002;21:101-13.

http://dx.doi.org/10.1016/S0167-9457(01)00057-4

26. Beaudoin N, Fleury J. Techniques de reeducation neuromusculaire appliqués à l' accidente vasculaire cerebral adulte. Encycl Més Chir 1995;26:455-63. 27.Intervenção fisioterapêutica nas disfunçôes do ombro em portadores de hemiparesia (endereço da internet). Paraná: Clementino e Leal (última atualizaçẫo: 03/2004; citado em: 01/2003). Disponível em: docente.fir.Br/copesq/ monografias/fi/2003_1. 